\title{
Prospective multicenter evaluation of rapid, gated SPECT myocardial perfusion upright imaging
}

\author{
Jamshid Maddahi, MD, FACC, ${ }^{a}$ Ricardo Mendez, MD, ${ }^{\mathrm{a}}$ John J. Mahmarian, MD, \\ Gregory Thomas, MD, ${ }^{\mathrm{c}}$ Hetal Babla, MS, ${ }^{\mathrm{d}}$ Chuanyong Bai, PhD, ${ }^{\mathrm{d}}$ Samia Arram, \\ BS, MBA, ${ }^{d}$ Peter Maffetone, CNMT, ${ }^{d}$ and Richard Conwell, BS $^{\mathrm{d}}$
}

Background. A novel three-dimensional (3D) iterative image reconstruction method (3DOSEM) has been developed that in phantom studies yielded comparable image quality at one half the imaging time. In this study, we compared standard (STD) and rapid (nSPEED) protocols for diagnostic quality of images and quantitation of end-diastolic volume (EDV), endsystolic volume (ESV), left ventricular ejection fraction (LVEF), and perfusion defect severity.

Methods. At 11 US community centers, 448 patients prospectively underwent rest-stressgated SPECT imaging using Tc-99m-labeled agent. The difference in quality and diagnostic equivalence of STD and nSPEED images were blindly evaluated by three experts. Defect intensity was quantitated as \% normal in the three coronary artery territories.

Results. Studies were abnormal in $40 \%$ of patients. In $98.7 \%$ of stress and $98 \%$ of rest images, the nSPEED image quality was identical to or better than the STD images. nSPEED images were diagnostically equivalent to the STD in $444 / 448(99 \%)$ patients. A high correlation was observed between nSPEED and STD studies for measurement of EDV $\left(Y=0.957 X, R^{2}=0.99\right)$, ESV $\left(Y=0.962 X, R^{2}=0.99\right)$, and LVEF $\left(Y=1.005 X, R^{2}=0.96\right)$. STD and nSPEED studies were not significantly different $(P=n s)$ for quantitative perfusion defect severity.

Conclusion. Rapid, gated rest-stress myocardial perfusion upright SPECT imaging may be achieved without compromising perfusion and function information. ( $J$ Nucl Cardiol 2009;16:351-7.) disease

Key Words: Myocardial perfusion imaging $\cdot$ SPECT $\cdot$ image processing $\cdot$ coronary artery

See related editorial, pp. 335-338

From the Departments of Molecular and Medical Pharmacology (Nuclear Medicine) and Medicine (Cardiology), ${ }^{\mathrm{a}}$ David Geffen School of Medicine at UCLA, Los Angeles, CA; Methodist DeBakey Heart Center, ${ }^{\mathrm{b}}$ The Methodist Hospital, Houston, TX; Mission Internal Medicine Group, ${ }^{\mathrm{c}}$ Mission Viejo, CA and Digirad Corporation, ${ }^{\mathrm{d}}$ Poway, CA.

Financial support for this study was partly provided by Digirad Corporation, Poway, CA.

Received for publication Sep 17, 2008; final revision accepted Jan 25, 2009.

Reprint requests: Jamshid Maddahi, MD, FACC, 100 UCLA Medical Plaza \#410, Los Angeles, CA 90095-7064; jmaddahi@mednet. ucla.edu.

$1071-3581 / \$ 34.00$

Copyright (C 2009 The Author(s). This article is published with open access at Springerlink.com.

doi:10.1007/s12350-009-9063-3
Recent technological developments in SPECT myocardial perfusion imaging (MPI) have focused on achieving higher imaging efficiency through rapid imaging. Benefits of rapid SPECT MPI include decreased patient motion and associated artifact, increased patient convenience and patient throughput, possibility of reducing the injected dose of perfusion tracer, and potential for dynamic imaging and absolute quantitation of myocardial blood flow.

Imaging efficiency may be enhanced by improving imaging system sensitivity so that one can acquire the same number of counts in a shorter period of time. ${ }^{1,2}$ Another approach is application of advanced image reconstruction algorithms so that one can obtain the same image quality with less acquired counts. Several such algorithms have been developed that include: (1) Use of iterative reconstruction algorithms such as MLEM and OSEM (instead of the filtered-back-projection algorithm) to better model and suppress image noise ${ }^{3}{ }^{3}$ (2) application of three-dimensional (3D) [instead of two-dimensional 
(2D)] image reconstruction for detector response modeling; and (3) iterative reconstruction algorithms with the modeling of other image degrading effects (such as attenuation, scatter, etc.). ${ }^{4}$ Such image quality improvement by using 3D reconstruction may allow one to reduce the acquisition time and maintain image quality as compared to using 2D reconstruction.

Bai et $\mathrm{al}^{5}$ have developed nSPEED 3D-OSEM algorithm that uses a slab-by-slab approach derived from the slice-by-slice incremental blurring model proposed by Zeng et al. ${ }^{6}$ In this approach, each slab that consists of several neighboring vertical slices goes through the convolution process rather than each vertical slice. This further reduces the number of convolutions and consequently the computational expense.

The purpose of the current study was to compare the results of the 3D-OSEM image reconstruction technique (nSPEED) to the standard (STD) 2D-OSEM iterative reconstruction in patients undergoing rest-gated stress SPECT myocardial perfusion studies with respect to (1) diagnostic quality of the images; (2) quantitative perfusion defect severity; and (3) measurement of left ventricular ejection fraction (LVEF), end-diastolic volume (EDV), and end-systolic volume (ESV).

\section{METHODS}

\section{Study Population}

A total of 448 patients were enrolled in this prospectively designed study from 11 US nuclear cardiology laboratories in community practices (Table 1). Patients underwent rest-gated stress SPECT imaging using a Tc-99mlabeled agent. All patients were referred for clinically indicated rest-stress myocardial perfusion SPECT imaging. There were 297 males (ranging in age from 30 to 89 years, mean $=67$ years) and 151 females (ranging in age from 25 to 95 , mean $=68$ years).

\section{Stress-Testing Procedures}

Patients underwent a symptom-limited treadmill exercise testing $(53 \%)$ or pharmacologic stress testing using infusion of adenosine $(37 \%)$, dipyridamole $(9 \%)$, or dobutamine $(1 \%)$. Treadmill exercise was performed using a modified or standard Bruce protocol. Heart rate, blood pressure, and 12-lead electrocardiograms were recorded before and during each minute of exercise. Patients were instructed not to consume caffeinecontaining products for 12 to 24 hours prior to pharmacologic stress testing with adenosine or dipyridamole. Adenosine stress testing consisted of 5 to 6 minutes of intravenous adenosine infusion $(0.140 \mathrm{mg} / \mathrm{kg} /$ minute $)$. Intravenous dipyridamole infusion was given for 4 minutes at $0.140 \mathrm{mg} / \mathrm{kg} /$ minute. Dobutamine infusion dose began at $5 \mu \mathrm{g} /$ minute and was increased, at 3-minute intervals, to 10,20 , and $40 \mu \mathrm{g} /$ minute.

\section{SPECT Procedures}

SPECT image acquisition was performed in accordance with American Society of Nuclear Cardiology guidelines and using the imaging protocol that was standard for each institution. Resting SPECT images were obtained about 30 to 45 minutes following I.V. injection of approximately 7 to $10 \mathrm{mCi}$ of Tc-99m-labeled agent. Injected dose varied based on patient's weight. Twenty to thirty $\mathrm{mCi}$ of Tc-99m-labeled agent was injected 60 to 90 seconds before termination of treadmill exercise, 2 to 3 minutes before termination of adenosine infusion, 3 minutes after termination of dipyridamole infusion, and 60 to 90 seconds before termination of dobutamine infusion. Stress-gated SPECT imaging began approximately 15 to 30 minutes after exercise and 45 to 90 minutes after termination of pharmacologic stressors.

Images were acquired with single head $(N=20)$, dual head $(N=239)$, or triple head $(N=189)$ Digirad cameras. All

Table 1. Participating centers

\begin{tabular}{lllll} 
No. & \multicolumn{1}{c}{ Site name } & \multicolumn{1}{c}{ Location } & $\begin{array}{c}\text { Representative } \\
\text { MD }\end{array}$ & Type of camera \\
\hline 1 & DIS Hanover & Hanover, MD & Multiple & Triple head \\
2 & Mexico Cardivascular Associates & Mexico, MO & Dr. L.R. Handlin & Dual head \\
3 & CardioSpecialists Group, Inc. & Munster, IN & Dr. R. Litchfield & Dual head \\
4 & DIS Orlando & Orlando, FL & Dr. P. Alagona & Single head \\
5 & DIS LIMA & St. Mary's, OH & Dr. S. Wolery & Dual head \\
6 & DIS Ontario & Montclair, CA & Dr. R. Ashwani Bahl & Single head \\
7 & Cardiology Associates of West Reading & Wyomissing, PA & Dr. R. Pattilo & Triple head \\
8 & Scripps Mercy Hospital & Chula Vista, CA & Dr. K.J. Van Lom & Triple head \\
9 & DIS Ft. Lauderdale & Lauderdale, FL & Multiple & Single head \\
10 & Kennestone Heart & Woodstock GA & Dr. D. Patel & Dual head \\
11 & Browhard Heart Specialists of Plantation & Plantation, FL & Multiple & Dual head \\
\hline
\end{tabular}


Table 2. Mean acquisition times (in minutes) for rest and stress studies for each camera type

\begin{tabular}{|c|c|c|c|c|c|c|}
\hline & \multicolumn{2}{|c|}{ C1 (single head) } & \multicolumn{2}{|c|}{ C2 (dual head) } & \multicolumn{2}{|c|}{ C3 (triple head) } \\
\hline & Rest & Stress & Rest & Stress & Rest & Stress \\
\hline Number of projections & 32 & 32 & 32 & 32 & 60 & 60 \\
\hline $\begin{array}{l}\text { Mean time/projection } \\
\text { Full-time (seconds) }\end{array}$ & 35 & 23 & 47 & 31 & 27 & 23 \\
\hline $\begin{array}{l}\text { Mean time/projection } \\
\text { Half-time (seconds) }\end{array}$ & 17.5 & 11.5 & 23.5 & 15.5 & 13.5 & 11.5 \\
\hline $\begin{array}{l}\text { Mean total acquisition } \\
\text { Time for full-time (minutes) }\end{array}$ & $\begin{array}{c}19.19 \pm \\
4.62\end{array}$ & $\begin{array}{l}13.00 \pm \\
2.39\end{array}$ & $\begin{array}{l}12.81 \pm \\
2.16\end{array}$ & $\begin{array}{c}8.39 \pm \\
2.64\end{array}$ & $\begin{array}{l}9.29 \pm \\
1.82\end{array}$ & $\begin{array}{l}8.05 \pm \\
1.76\end{array}$ \\
\hline Mean total acquisition & $9.85 \pm$ & $6.76 \pm$ & $6.53 \pm$ & $4.32 \pm$ & $4.81 \pm$ & $4.18 \pm$ \\
\hline Time for half-time (minutes) & 2.31 & 1.02 & 1.08 & 1.32 & 0.91 & 0.88 \\
\hline
\end{tabular}

Note that the mean total acquisition time for half-time is not exactly $1 / 2$ of that for full-time acquisition because chair motion times are similar for both imaging methods ( 0.52 minutes for $\mathrm{C} 1,0.25$ minutes for $\mathrm{C} 2$, and 0.32 minutes for $\mathrm{C} 3)$. Mean total acquisition times were significantly $(P<0.05)$ shorter for the half-time (nSPEED) method as compared to the full-time (STD) method for all three camera types.

images were simultaneously acquired at full-time and halftime. Acquisition times for each type of camera are shown in Table 2. SPECT images were reconstructed into short axis, vertical long axis, and horizontal long axis planes. Images were processed using Digirad's 2D-OSEM and nSPEED reconstruction techniques on Mirage Processing software.

There are several different approaches to detector response modeling for $3 \mathrm{D}$ reconstruction. An effective and efficient one is the slice-by-slice incremental blurring model proposed by Zeng et al. ${ }^{6}$ In this technique, a vertical slice of the reconstructed volume that is parallel to the detector surface is only convolved with a very small kernel (such as a $3 \times 3$ kernel) and the result is added to the next vertical slice, which is closer to the detector surface. The result in the next vertical slice is then convolved with another small kernel and then added to the next vertical slice and so on. The small kernel is calculated from the different detector response functions of the two neighboring vertical slices. This model significantly reduces the computational expense compared to non-incremental convolution-based approaches.

Butterworth post-filters were applied to the volume images. Butterworth cutoff frequency and order were, respectively, 0.37 and 6 for non-gated rest and summed stress images and were 0.33 and 6 for gated stress images. LVEF, EDV, and ESV were measured using Cedars-Sinai's QGS software. ${ }^{7}$ Defect intensity within the left anterior descending (LAD), left circumflex (LCX), and right coronary artery (RCA) territories were calculated by averaging the computer-generated segmental counts (expressed as \% maximum) for all segments within each coronary territory.

Rest and stress image sets for the full-time and nSPEED acquisition methods were visually scored by consensus opinion of three experienced observers (Jamshid Maddahi, MD; John J. Mahmarian, MD; and Gregory Thomas, MD), who were blinded to the type of study and patient data. STD and nSPEED images were randomly displayed on the left or right reading stations side-by-side and were compared for image quality and diagnostic equivalence. Image quality was scored separately for stress and rest images using a 4-point scoring system: $1=$ left identical to right, $2=$ left slightly better than right, $3=$ left mildly better than right, and $4=$ left significantly better than right. Diagnostic equivalence was scored for the rest-stress image sets using a 3-point scoring system: 1 = diagnostically equivalent, $2=$ possible difference in interpretation, and $3=$ probable difference in interpretation.

\section{Statistical Analyses}

Defect intensities were compared using Paired $t$-test (with two sample means) analysis in Microsoft Excel. Relation between the STD and nSPEED values for EDV, ESV, and LVEF was assessed by linear regression analysis. A BlandAltman assessment for agreement was used to compare the nSPEED and STD imaging methods for quantitation of perfusion defect sizes and measurement of EDV, ESV, and LVEF. A range of agreement was defined as mean bias \pm 2 SD.

\section{RESULTS}

By consensus visual analysis, 269/448 (60\%) of the studies were normal and the remaining 177/448 (40\%) were abnormal. The proportion of normal and abnormal studies is representative of that generally encountered in office-based nuclear cardiology practices. Body surface area of the study population ranged from 1.40 to $2.80 \mathrm{~m}^{2}$ $\left(2.01 \pm 0.26 \mathrm{~m}^{2}\right)$.

\section{Blinded Visual Assessment of Image Quality}

In $79.5 \%(356 / 448)$ of stress and $78.6 \%(352 / 448)$ of rest images, the visual score was found to be identical (score of 1). In 19.2\% (86/448) of stress and 19.4\% (87/ 448 ) of rest images, the nSPEED image quality was 
scored to be slightly or mildly better than (scores of 2 or 3 ) the STD images. In $1.3 \%(6 / 448)$ of stress and $2 \%$ (9/448) of rest images, the STD image quality was scored to be slightly or mildly better than that of the nSPEED. Therefore, a significantly higher percentage of studies (stress: $19.2 \%$ vs $1.3 \%, P<0.01$ and rest: $19.4 \%$ vs $2 \%, P<0.01)$ were judged to have a better image quality by nSPEED vs the STD full-time method.

\section{Diagnostic Equivalence Between nSPEED and Full-Time Acquisition}

Of the $448,444(99 \%)$ of the stress images and 444 $(99 \%)$ of the rest images were scored as diagnostically equivalent by blinded visual analysis.

\section{Quantitation of Perfusion Defect Intensity on Gated Stress and Rest Images}

As shown in Figures 1 and 2, quantitative perfusion defect intensities on rest images in the left anterior descending (LAD), left circumflex (LCX), and right coronary arteries (RCA) were, respectively, 74\%, 72\%, and $63 \%$ for nSPEED images which were not significantly different than those of the STD images which were, respectively, 76\%, 74\%, and 66\%. Furthermore, quantitative perfusion defect intensities on stress images in the LAD, LCX, and RCA territories were $76 \%, 73 \%$, and $64 \%$ for nSPEED images which were not significantly different than those of the STD images which were, respectively, $77 \%, 74 \%$, and $66 \%$. Table 3 demonstrates that stress and rest quantitative perfusion defect intensities were not significantly different between nSPEED and STD images in any of the three coronary territories in the exercise and pharmacologic stress subgroup of patients. Table 4 shows the results of Bland-Altman analysis for agreement between nSPEED and STD methods for quantitation of stress and rest perfusion defect sizes in the three coronary arteries. The range for $95 \%$ limits of agreement between the two methods was small and clinically acceptable.

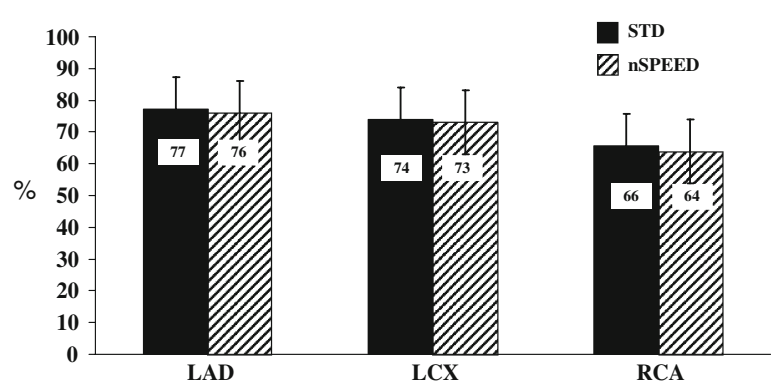

Figure 1. Gated stress quantitative perfusion defect intensities: nSPEED vs STD.

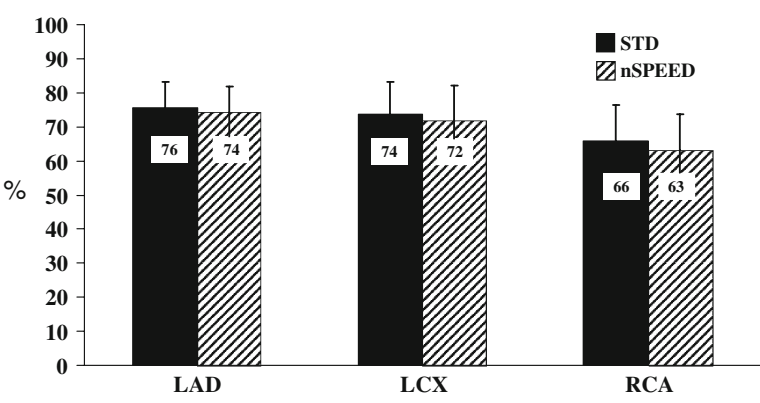

Figure 2. Rest quantitative perfusion defect intensities: nSPEED vs STD.

\section{Measurement of LVEF, EDV, and ESV from Gated Stress Images}

A very high correlation was observed between nSPEED and STD acquisition for measurement of EDV $\left(Y=0.957 X, \quad R^{2}=0.99\right), \quad \mathrm{ESV} \quad(Y=0.962 X$, $\left.R^{2}=0.99\right)$, and LVEF $\left(Y=1.005 X, R^{2}=0.96\right)$. Correlations were equally high in exercise and pharmacologic stress patient subgroups (Figures 3-5). Figures 6, 7, and 8, respectively, display Bland-Altman plots for EDV, ESV, and LVEF measurements. These analyses indicate that the mean difference and range for the $95 \%$ limits of agreement between nSPEED and STD images were small and clinically insignificant.

\section{Case Illustration}

Figure 9 demonstrates (A) STD and (B) nSPEED SPECT images with identical partially reversible inferolateral as well as reversible distal anteroseptal and apical defects. Quantitative results were also very similar. Defects intensities in the LAD, LCX, and RCA territories for the STD stress images were, respectively, $68 \%, 66 \%$, and $56 \%$ and for the rest images were, respectively, 77\%, 71\%, and 58\%. For STD images, EDV was $64 \mathrm{~mL}$, ESV was $25 \mathrm{~mL}$, and LVEF was $62 \%$. Defect intensities in the LAD, LCX, and RCA territories for the nSPEED stress images were, respectively, 68\%, 67\%, and 55\% and for the rest images were, respectively, $76 \%, 72 \%$, and $56 \%$. For nSPEED images, EDV was $58 \mathrm{~mL}$, ESV was $22 \mathrm{~mL}$, and LVEF was $61 \%$.

\section{DISCUSSION}

In this study, we observed a very high qualitative and quantitative correlation between nSPEED and STD SPECT images. As the number of iterations of the OSEM algorithm and choice of post-filters in nSPEED will influence image results, in a separate pilot study we had optimized the nSPEED package to make the 
Table 3. Subgroup analyses of stress and rest quantitative perfusion defect intensities in the exercise and pharmacologic stress subgroup of patients

\begin{tabular}{|c|c|c|c|c|c|c|}
\hline & \multicolumn{3}{|c|}{ Stress } & \multicolumn{3}{|c|}{ Rest } \\
\hline & LAD & LCX & RCA & LAD & LCX & RCA \\
\hline \multicolumn{7}{|l|}{ Exercise } \\
\hline STD & $78 \pm 8$ & $74 \pm 11$ & $66 \pm 11$ & $76 \pm 7$ & $73 \pm 9$ & $66 \pm 10$ \\
\hline nSPEED & $76 \pm 8$ & $73 \pm 11$ & $64 \pm 11$ & $75 \pm 7$ & $71 \pm 10$ & $63 \pm 10$ \\
\hline \multicolumn{7}{|c|}{ Pharm stress } \\
\hline STD & $77 \pm 8$ & $74 \pm 11$ & $65 \pm 11$ & $75 \pm 8$ & $74 \pm 10$ & $65 \pm 11$ \\
\hline nSPEED & $76 \pm 8$ & $73 \pm 11$ & $63 \pm 11$ & $74 \pm 8$ & $72 \pm 10$ & $63 \pm 11$ \\
\hline
\end{tabular}

Results were not significantly different between nSPEED and STD images in any of the three coronary territories.

Table 4. Results of Bland-Altman analysis for agreement between nSPEED and STD methods for quantitation of stress and rest perfusion defect intensities in the three coronary arteries

\begin{tabular}{|c|c|c|c|c|c|c|}
\hline & \multicolumn{3}{|c|}{ Stress } & \multicolumn{3}{|c|}{ Rest } \\
\hline & LAD & $\mathbf{L C X}$ & RCA & LAD & $\mathbf{L C X}$ & RCA \\
\hline Mean & 1.26 & 0.91 & 1.71 & 1.31 & 1.89 & 2.77 \\
\hline Limits of agreement $(+2 \sigma)$ & 6.20 & 9.04 & 9.20 & 7.11 & 11.85 & 12.29 \\
\hline Limits of agreement $(-2 \sigma)$ & -3.68 & -7.22 & -5.79 & -4.49 & -8.06 & -6.75 \\
\hline Outliers & $4 \%$ & $4 \%$ & $6 \%$ & $3 \%$ & $7 \%$ & $6 \%$ \\
\hline
\end{tabular}

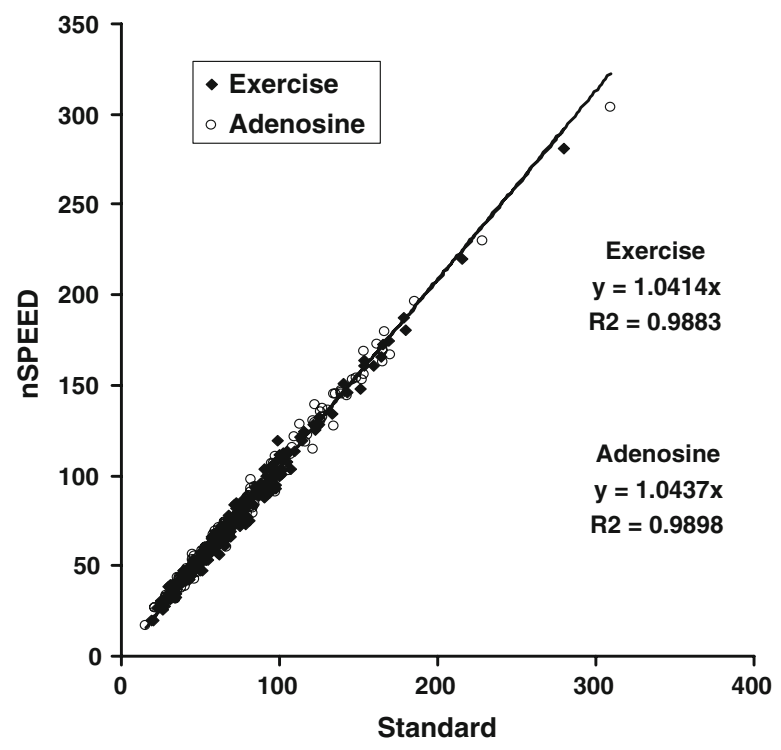

Figure 3. Measurement of EDV (mL) from gated stress images: nSPEED vs STD.

nSPEED half-time images equivalent to the conventional 2D-OSEM full-time images, both qualitatively and quantitatively.

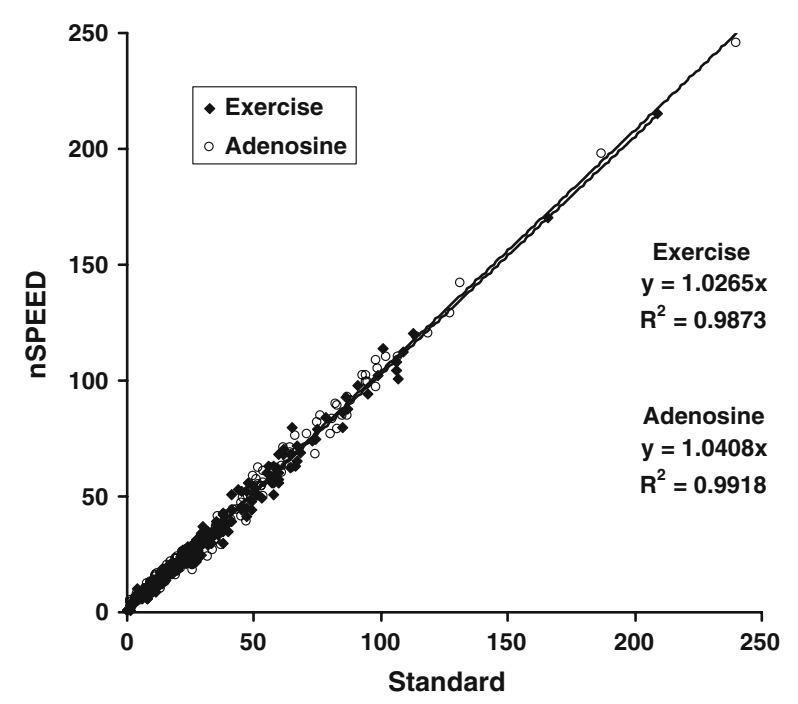

Figure 4. Measurement of ESV (mL) from gated stress images: nSPEED vs STD.

In this study, for nSPEED reconstruction, Butterworth cutoff frequency and order were, respectively, 0.37 and 6 for non-gated rest and summed stress images and were 0.33 and 6 for gated stress images. We have observed a systematic underestimation of EDV and ESV 


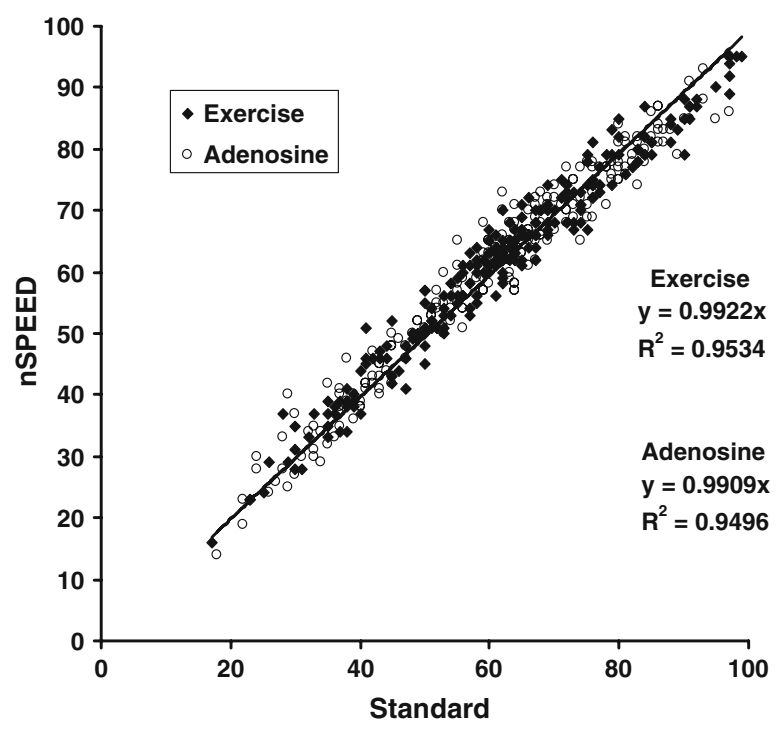

Figure 5. Measurement of LVEF from gated stress images: nSPEED vs STD.

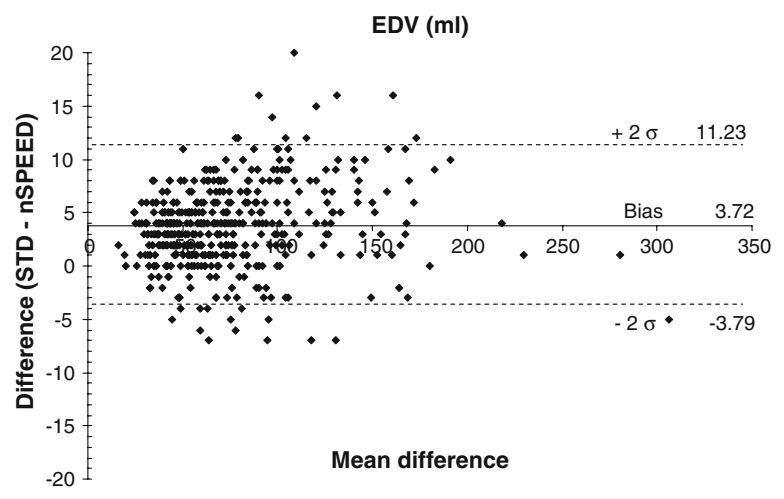

Figure 6. Bland-Altman plots for measurement of EDV by nSPEED and STD methods.

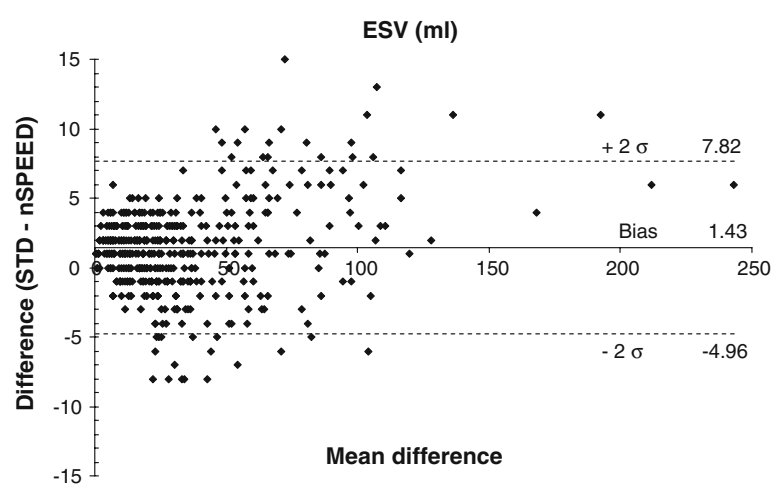

Figure 7. Bland-Altman plots for measurement of ESV by nSPEED and STD methods.

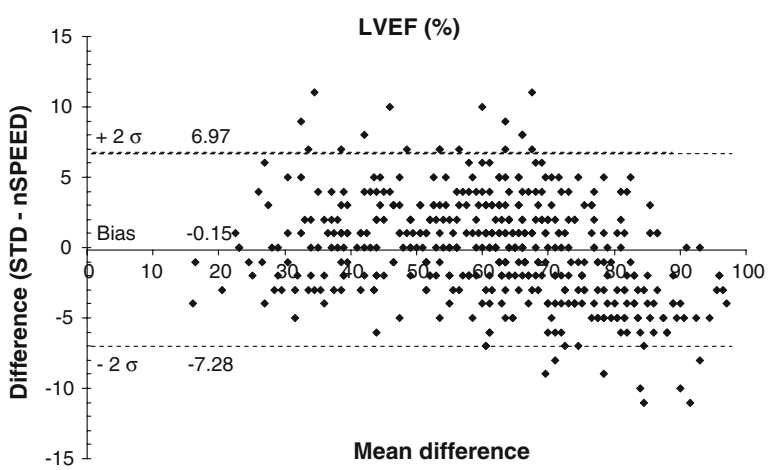

Figure 8. Bland-Altman plots for measurement of LVEF by nSPEED and STD methods.

as well as overestimation of LVEF when Butterworth cutoff frequency of 0.33 and order of 6 were compared to Butterworth cutoff frequency of 0.23 and order of 6 for post-filtering of gated stress images. ${ }^{8}$ We have also noticed that use of 4D MAP or optical flow reconstruction for noise suppression of gated images might result in significantly lower LVEF values. ${ }^{8}$

In addition to the nSPEED software developed by Digirad, there are other 3D reconstruction software packages that also claim the capability of allowing shortened scanning time while maintaining or improving image quality and quantitation. These techniques include the Wide-Beam Reconstruction (WBR) from UltraSPECT, ${ }^{9,10}$ the Flash-3D from Siemens, Astonish from Philips, and Evolution from GE. The physics principle of all the 3D reconstruction packages is the same, i.e., to model the 3D depth-dependent detector spatial response and to suppress the noise to achieve the best compromise of resolution/contrast improvement and noise reduction. The differences between these software packages are related to how the modeling of detector spatial response is implemented and how the noise reduction is performed. Technical papers are not yet published on these methods to reveal the details of the spatial response modeling and noise reduction approaches. Technical notes from Philips on Astonish only mention that Astonish uses matched filters, and UltraSPECT only mentions that WBR does not need post-filtering as images are filtered in the WBR reconstruction.

\section{CONCLUSION}

This study demonstrated that, as compared to 2D-OSEM iterative reconstruction, 3D-OSEM image reconstruction technique allows reduction of imaging time to one half and yields diagnostically equivalent images for rest and stress studies, reliable measurement of EDV, ESV, and LVEF from gated stress SPECT 

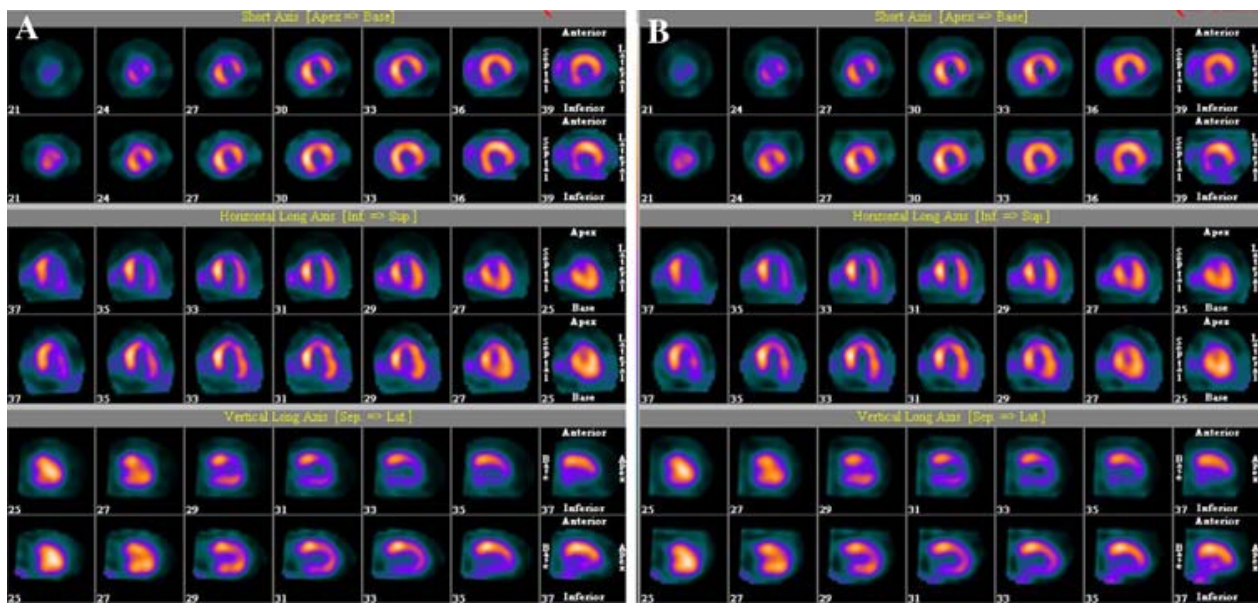

Figure 9. A, Standard and B, nSPEED SPECT images show identical partially reversible inferolateral as well as reversible distal anteroseptal and apical defects. Quantitative results were also very similar (please see the main text).

images, and reliable quantitation of perfusion defect severity on stress and rest SPECT images.

\section{Open Access}

This article is distributed under the terms of the Creative Commons Attribution Noncommercial License which permits any noncommercial use, distribution, and reproduction in any medium, provided the original author(s) and source are credited.

\section{References}

1. Funk T, Kirch DL, Koss JE, Botvinick E, Hasegawa BH. A novel approach to multipinhole SPECT for myocardial perfusion imaging. J Nucl Med 2006;47:595-602.

2. Sharir T, Ben-Haim S, Merzon K, Prochorov V, Dickman D, BenHaim S, et al. High-speed myocardial perfusion imaging. Initial clinical comparison with conventional dual detector Anger camera imaging. J Am Coll Cardiol Imaging 2008;1:156-63.

3. Bruyant PP. Analytic and iterative reconstruction algorithms in SPECT. J Nucl Med 2002;43:1343-58.

4. Tsui BM, Frey EC, LaCroix KJ, Lalush DS, McCartney WH, King MA, et al. Quantitative myocardial perfusion SPECT. J Nucl Cardiol 1998;5:507-22.
5. Bai C, Zeng GL, Gullberg GT, DiFilippo F, Miller S. Slab-by-slab blurring model for geometric point response correction and attenuation correction using iterative reconstruction algorithms. IEEE Trans Nucl Sci 1998;45:2168-73.

6. Zeng GL, Gullberg GT, Bai C, Christian PE, Trisjono F, DiBella EVR, et al. Iterative reconstruction of fluorine-18 SPECT using geometric point response correction. J Nucl Med 1998;39: 124-30.

7. Germano G, Kiat H, Kavanagh PB, Moriel M, Mazzanti M, Su $\mathrm{H}$, et al. Automatic quantification of ejection fraction from gated myocardial perfusion SPECT. J Nucl Med 1995;36: 2138-47.

8. Babla H, Bai C, Agili S, Conwell RL, Maddahi J. Effect of postfilters on left ventricular ejection fraction for gated cardiac SPECT studies. J Nucl Cardiol 2008;15:S20. (abstract).

9. Borges-Neto S, Pagnanelli RA, Shaw LK, Honeycutt E, Shwartz SC, Adams GL, et al. Clinical results of a novel wide beam reconstruction method for shortening scan time of Tc-99m cardiac SPECT perfusion studies. J Nucl Cardiol 2007;14:555-65.

10. DePuey EG, Gadiraju R, Clark J, Thompson L, Anstett F, Shwartz SC. Ordered subset maximization and wide beam reconstruction "half-time" gated myocardial perfusion SPECT functional imaging: A comparison to "full-time" filtered backprojection. J Nucl Cardiol 2008;15:547-63. 